\title{
Color Representation of Electron Microprobe Area-Scan Images by a Color Separation Process
}

\author{
Harvey Yakowitz and Kurt F. J. Heinrich \\ Institute for Materials Research, National Bureau of Standards, Washington, D.C. 20234
}

(August 9, 1968)

\begin{abstract}
Composite color photographs were prepared using x-ray area scanned images from the electron probe microanalyzer. Three-color composites are completely interpretable in terms of the primary color (red, green or blue) chosen to represent each element. The color pictures were obtained by preparing conventional black-and-white scanning images which were then used as color-separationpositives, with appropriate filters, to make color prints. Methods for preparing and interpreting color composites are considered. Specifically, color mixing, proper choice of filters to match film characteristics, exposure criteria, and choice for each partial image are discussed in detail. Finally, typical applications drawn from the fields of metallurgy, mineralogy, and biology are shown to illustrate the principles described. With the use of Polaroid film this procedure is quite convenient.
\end{abstract}

Key words: Color photography; metallography; petrography; photomicrography; scanning electron microprobe; $\mathrm{x}$-ray microscopy.

\section{Introduction}

The scanning electron probe microanalyzer $[1]^{1}$ can produce images of microscopic areas as a function of the emission of $x$-rays or of electrons. The various electron images provide mainly topographic information, while the x-ray images show the spatial distribution of the elements present in the specimen. Thus, the scanning electron probe is a microscope as well as an $\mathrm{x}$-ray spectrometer [2].

Black-and-white photographs showing the topographic distribution of a single signal are widely used. It is difficult, however, to show the correlation of signals from two or more x-ray lines, or of x-ray signals, with the topography provided by the electron images, without the use of color. Therefore, several investigators have proposed color photography for this purpose and some examples have been shown [3-6].

This paper describes the results of a systematic study of color composite micrographs. In discussing the results, emphasis will be placed on the interpretation of the composite color micrograph.

\section{General Methods for the Preparation of Color Composites}

Color micrographs of x-ray area scans can be obtained in three ways:

1. By the use of a three-gun color cathode ray tube (CRT) and color photography of the three signals generated simultaneously [6].

2. By using a single-gun cathode ray tube with color filters interposed between the screen and the color film in the oscilloscope camera. In this case, a phosphor

${ }^{1}$ Figures in brackets indicate the literature references at the end of this paper. of broad spectral distribution is used; and the three individual signals are recorded sequentially, changing filters between exposures [5].

3. By preparing conventional black-and-white scanning images and using them as color-separationpositives, with appropriate filters, to make color prints $[3,4]$. We shall call this method the colorseparation method.

The first method (which we have not used) is the only one permitting immediate simultaneous viewing of the signals in their respective colors. As many as three signals can be recorded simultaneously. This method requires, however, the addition of fairly expensive and massive equipment.

The second method requires only minor modifications if the conventional equipment for area scans is available. In this method, the various signals must be sequentially recorded. Since the color composite is not visually displayed, this technique is tedious unless rapid access photography, such as the Polaroid process is used. However, with the currently available films, and the low brightness and limited spectral range of oscilloscope-tube phosphors, the recording of x-ray pulse signals is difficult. Furthermore, if any of the three exposures is incorrect, the entire procedure must be repeated.

The third method also requires that the signals be recorded sequentially (unless several oscilloscopes are provided with cameras). However, the exposures can be made by the usual technique for black-andwhite scanning photographs. As shown here, readily interpretable color composites can be produced routinely, with the aid of a camera equipped with a Polaroid back. No large expenditures for equipment are necessary. 
The second and third methods were investigated and reported upon in a paper by Jones, Gavrilovič, and Beaven [7]. These authors concluded that the method of preparing composites directly on the microprobe (second method) was more satisfactory than the color-separation method. We have investigated both methods in detail and have arrived at the conclusion that the color-separation method is the most advantageous.

The color-separation technique can be easily used on a routine basis, by unskilled personnel, with a minimum of training. Another important advantage is the possibility of deciding upon the best color combination after the individual black-and-white pictures have been taken. The yield can be 15 to 20 color composites of uniform quality per hour. In view of the advantages of this technique, the work presented in this paper was performed by means of the color-separation technique.

The color photograph is prepared as follows:

1. The appropriate $\mathrm{x}$-ray and electron signal scans are prepared as positive black-and-white prints by photographing the cathode ray tube of the microprobe in the usual fashion [6].

2. The separation-positives are sequentially photographed by the copying camera (which is loaded with Polaroid color film); for each partial exposure, a different color filter is placed between the camera lens and the separation-positive.

The success of this technique depends upon: (1) the quality of the black-and-white images, (2) the characteristics of the light source, the film and color filters, (3) the exposure times, (4) the proper choice of color for each partial image, and (5) avoiding the relative displacement of the black-and-white images during the color exposure. Before discussing each of these requirements, we must consider the effect of mixing two or more colors. This consideration is of prime importance for the interpretation of the final color picture.

\section{Aspects of Additive Color Mixing}

In color reproduction one obtains a wide range of colors by mixing relatively few basic primary colors in varying proportions. Red, green, and blue are customarily chosen as primary colors for additive mixing because mixing them produces a wide range of colors. None of the primaries can be obtained from mixing the other two. Hence, the presence or absence of primaries can be ascertained in color mixtures if these three primaries are chosen. Almost any color can be made by a mixture of red, green, and blue light in a particular proportion which then, in principle, defines uniquely this nonprimary color. This includes white, gray or black which thus may be considered to be a mixture of red, green and blue. Due to the characteristics of the human eye, we cannot unequivocally interpret mixtures of more than three components. Therefore, we should not have more than three primary colors in one system.
Color film is based upon the same primaries - red, green, and blue. Like the eye, the color film can only resolve mixtures of three primaries. Thus, only three primaries can be used if any color produced by mixing in the composite is to be interpreted in terms of the primaries. For example, let iron on the separationpositive be made red and nickel on the second separation be made blue. If an $\mathrm{Fe}-\mathrm{Ni}$ alloy is present on the composite, a red-blue mixture (magenta) occurs. All magenta regions on the composite can then be interpreted as resulting from the simultaneous presence of $\mathrm{Fe}$ and $\mathrm{Ni}$. Using more than three primary colors $[7,8]$ leads to color mixtures of the same chromaticity (hue and saturation) for more than one combination of elements. On the other hand, if color is only being used to identify a phase, as in coloring a map, then as many colors as there are phases can be used-color mixtures will then have no clear meaning in terms of composition. If, for instance, only discrete elements (with no mixing) occur, then as many colors as there are elements can be used, in principle, to show the structural relationships in the specimen.

The process of exposing color composites consists of adding primaries since each successive filter color is stored on the color film. The three primary colors add to make white. The intermediate colors are: magenta (pinkish) which can be considered as red plus blue, or white minus green, yellow which is red plus green, or white minus blue, and cyan (sky color) which is blue plus green, or white minus red. The color resulting from omitting a single primary from white is called the complement of that primary.

The x-ray and electron micrographs used may contain all shades of gray. They are almost never pure black-and-white. For preparing composite color photographs, the ternary mixtures of the primaries resulting from such black-and-white pictures must be considered.

The ternary mixtures can be shown by the following method: A standard 16-step logarithmic gray scale is prepared and cut into an equilateral triangle having white as an apex and black along a base. The triangle is successively photographed with a red, green, and blue filter, having been rotated $120^{\circ}$ between each exposure. The gray scale is shown in figure 1 and the color triangle is seen in figure 2 (see color plate 1$){ }^{2}$. The primary colors appear at the apices. The binary mixtures yellow, magenta, and cyan can be observed in the center of each corresponding side of the triangle. Ternary color mixtures (including gray) are in the interior of the triangle.

Any mixed color in the picture can always be associated with its component primaries. However, quan-. titative interpretation of the color picture in terms of the composition corresponding to each point on the picture is impractical. This is because of the rigid standardization and control of the pictorial process which would be required starting with the black-andwhites on through a colorimetric reduction of the color data from the picture. Quantitative raster scanning

${ }^{2}$ Color plates 1,2, and 3 appear at the end of the paper. 


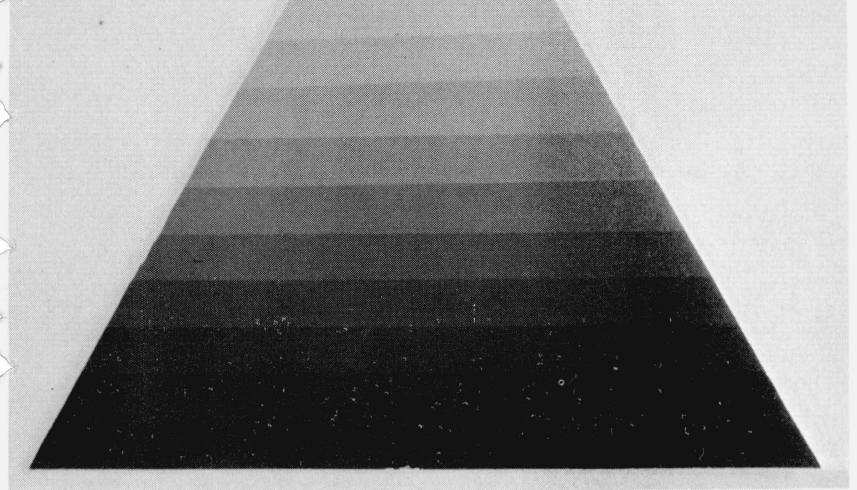

FiguRE 1. Triangular logarithmic 16-step grey scale used for color balancing.

[9] is a much simpler and more accurate means of obtaining quantitative information in digital form from a selected region of the specimen. For this reason, only qualitative information can be obtained from the picture, i.e., whether one, two, or three elements occur at the same physical place in the microstructure. Of the primary colors (figure $2-$ plate 1 ), red attracts the attention more so than green which in turn has a higher attention value than blue. Of the binary mixtures, yellow tends to be lighter than magenta and cyan. Hence, for pure elements which we wish to emphasize, red is a good choice while for mixtures requiring emphasis, yellow is often a good choice.

Much of the color mixing in a typical picture is binary because of the nature of most microprobe specimens. Thus, the binary color mixing occurring along the bases of the color triangle is worth a detailed examination. For this purpose, a double-length strip from the 16-step gray scale was photographed successively with each pair of primary color filters after being rotated $180^{\circ}$ between exposures. The results show that yellow is easily distinguished from both red and green-more so than magenta and cyan from their respective primary neighbors.

The relations and contrasts of colors obtainable from three primaries can be better illustrated by the following representation. Consider the color triangle discussed previously with the pure primaries at the apices. In the center we find neutral gray. However, since we distinguish a lightness scale from white to black, a vertical lightness scale can be represented on a perpendicular to the color triangle. Connecting the extremes (black and white) to the three primaries, we obtain a triangular bipyramid, figure 3 , which is similar to the Munsell color solid [10]. However, on this diagram, the horizontal plane represents the same lightness of the black-and-white pictures rather than the color picture. We have marked on this figure rela-

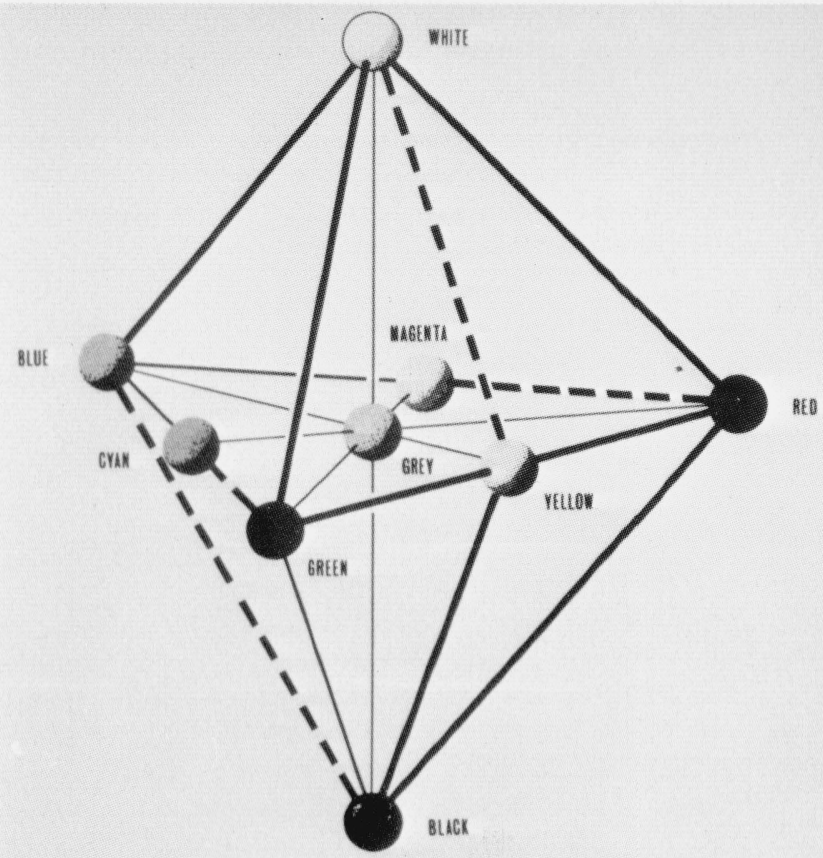

FIGURE 3. Schematic representation of the color solid pertaining to the red, green, blue primary system.

Highly contrasting pairs of colors are connected by bold solid lines, intermediate contrast is indicated by solid lines and poor contrast is denoted by broken line.

tions of high contrast by solid lines and poor contrast by broken lines. This graph may be helpful in color selections.

Figure 2 (plate 1) was prepared using a particular set of red, green and blue filters. Since other primary filters could have been used, the color triangle of figure 2 is a particular case among many. Choosing a particular filter set defines the gamut for a given film. The filters chosen for the color-separations should be picked so as to yield the widest gamut.

Proper balance of color on the film for a given set of filters is achieved when the addition of equal lightnesses on the black-and-white pictures yields a neutral gray on the color picture. This criterion was satisfied by obtaining a color picture of the unrotated gray scale, adjusting the exposure time for each filter until the photograph showed neutral gray steps. Changing the exposure time for a given filter changes the color balance. The light sources we used were incandescent photofloods. We also used cold white fluorescent tubes.

There are at least three sets of filters which can be properly balanced and which yield good color quality when using Polaroid film. These sets are standard Kodak Wratten series numbers 25, 58, 47, numbers 92, 93, 94, and numbers $33,93,45$ for red, green and blue respectively. The first set is that used to match the dye peaks of most sheet color films, the second was recommended by the film manufacturer and the third we found after some experimentation. The exposure times for each filter set are shown in table 1. With a variety of input subjects, the $33,93,45$ set provided the most satisfactory results. The mixed colors could 
easily be interpreted and color quality was good. This set was used to prepare all the illustrations in this paper.

TABLE 1. Exposure times for color composites*

Light source: Four reflector flood lamps spaced $2 \mathrm{ft}$ apart, $3 \mathrm{ft}$ from subject. Camera $20 \frac{1}{4}$ in from subject. Lens setting f5.6.

\begin{tabular}{cll}
\hline $\begin{array}{c}\text { Kodak Wratten filter } \\
\text { set number }\end{array}$ & Color & Time (sec.) \\
\hline 92 & Red & $1 / 4$ \\
93 & Green & 12 \\
94 & Blue & 60 (add $1 / 30$ white) \\
& & $1 / 8$ \\
25 & Red & $5 / 8$ \\
58 & Green & 5 \\
47 & Blue & $1 / 5$ \\
33 & Red & 10 \\
93 & Green & 3 (add $1 / 60$ white) \\
45 & Blue &
\end{tabular}

Light source: Two cold white fluorescent bulbs 15 in long placed 18 in from subject. Camera $3 \frac{1}{2}$ in from subject. Lens setting f5.6.

$\begin{array}{ll}33 & 1.5 \\ 93 & 40 \\ 45 & 7.5 \text { (add } 1 / 60 \text { white) }\end{array}$

*Included as a guide only. These values give approximately equal lightness.

Using the 33, 93, 45 set of filters, blue is sometimes difficult to distinguish from black. The lightness of blue can be increased by adding a small amount of white light to the blue subject. One exposes a subject with the aid of the blue filter and then reexposes the same subject for a much shorter time without a filter.

For dark separation-positives, the exposure time should be increased. This upgrades the dark separation-positive at the expense of the overall color balance of the composite. Exposure adjustment to minimize the effect of separation-positives of low quality is, however, a poor method which should be avoided.

\section{Preparation of Black-and-White Images}

In this paper we have used the conventional x-ray images, in which every detected pulse is shown as a white dot on black background. Rate-meter output images [2] can also be used in a similar fashion.

The first requirement for a satisfactory color composite is a set of x-ray black-and-white images of good quality. The main points to observe are:

1. The areas of high x-ray emission should be white, and those with no exposure, black. The effects of a gray background (e.g., through too short development time of the black-and-white picture) cannot be easily corrected in the making of the composite.

2. The interpretation of the color picture is often aided if equal lightness of the separation-positives corresponds to equal concentrations of the elements represented.

3. A sufficient amount of x-ray counts should be recorded [2].
4. Care should be taken that the specimen position with respect to the scan does not change between exposures, due to electrostatic charges or mechanical displacement.

\section{Registration Device}

A simple device provides entirely satisfactory alinement of the partial images. The separation-positives are located within a holder by means of several dial pins. By means of drilling the bed of the aluminum top plate with some holes, cementing the top and bottom plates and evacuating the assembly, one can hold the picture flat.

\section{Choice of Color for Each Partial Image}

Since the primaries and binary mixtures of the primaries differ inherently in attention-value, the choice of color for each constituent can be used to direct our attention to selected features of the composite. Thus, in figure $4 \mathrm{a}$, plate 1 the yellow area stands up markedly against the dark blue background.

An extreme case occurs where small areas of an x-ray signal are superposed upon the target current, backscatter, or even an x-ray image used as topographic background. In this case, the localized x-ray signal should be shown white (or yellow). A fairly dark color, such as green or cyan makes a satisfactory background. Since the lightness range of the background should be small but distinct, it might be difficult to produce the contrast within the background necessary for orientation. From this viewpoint blue is not very useful. Small areas of high lightness in the separation-positive for the background must be avoided. If these areas are toned down to the maximum lightness tolerable in the background, the remaining background topography will be lost. It is therefore advisable to overexpose the background separation-positive so that a considerable fraction of it attains maximum lightness. On toning down the lightness level for background, this will be the only remaining background information.

Different colors - primary as well as others - possess different degrees of attention value. Hence, the viewer's attention can be directed to various features of the color picture by a careful selection of the primary color used to represent each constituent. As an example figure 5 (see plate 2 for the $\mathrm{d}, \mathrm{e}, \mathrm{f}, \mathrm{g}, \mathrm{h}$, and i parts of this figure) shows three typical x-ray separation-positives and all six possible color composites obtainable from them. As the color combinations change the prominence of certain features including background changes.

\section{Applications}

The optical photomicrograph shown in figure $6 \mathrm{a}$ is a portion of a crack located near the edge of a specimen of a Ni base superalloy. The crack appeared during service at high temperature in air. The optical microscopist can distinguish two shades of gray in the crack. The microprobe identified $\mathrm{Al}$ and $\mathrm{Cr}$ in the 

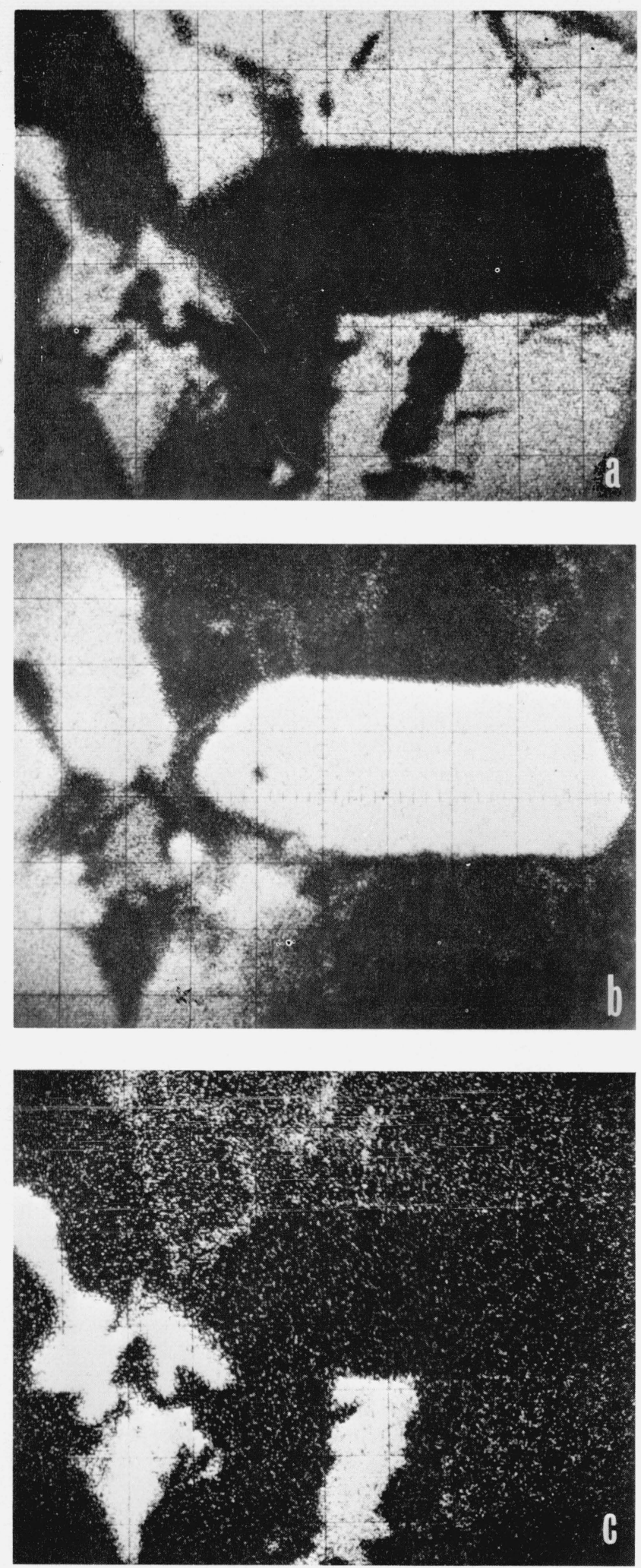

FIGURE 5. X-ray separation-positives of the naturally occurring mineral, galena, used for illustrating effects of color choice for each partial image.

a. $\mathrm{S}-\mathrm{K} \alpha . \quad$ b. $\mathrm{Si}-\mathrm{K} \alpha . \quad$ c. $\mathrm{Ag}-\mathrm{L} \alpha$. crack, and the locations of these elements as well as $\mathrm{Ni}$ are shown by the $\mathrm{x}$-ray area scans (figs. 6b, c, d). Working with only the $\mathrm{x}$-ray area scans, a good deal of patience and intuition is required to reconstruct the exact phase positions in the microstructure. Furthermore, the locations of superposed elements are difficult to discern. The color composite with Al red, $\mathrm{Cr}$ green, and $\mathrm{Ni}$ blue (fig. 6e, plate 3) shows exactly the phase positions in the microstructure. Mixed colors indicate the presence of superposed elements. The composite is topographically analogous to the optical micrograph, but the major constituents responsible for the structure are identified. The conclusion of the metallurgists regarding the specimen was that oxides of $\mathrm{Cr}$ and $\mathrm{Al}$ formed during service. These oxides are larger in volume than the host lattice and the $\mathrm{Ni}$ lattice failed. Crack propagation then proceeded as more oxide formed. The composite indicates that $\mathrm{Cr}$ oxidizes rapidly and that after most of the available $\mathrm{Cr}$ is oxidized, Al oxidizes. This mechanism is illustrated by the presence of the $\mathrm{Al}$ shell around the $\mathrm{Cr}$.

A typical set of input area scans of a heated dental alloy is shown in figure 7. Exact element superposition is difficult to ascertain without carefully measuring each picture-a tedious, time-consuming task. The composite figure 7d, plate 3 shows Ag-Sn signal superposition yellow. In addition, there are a few particles containing $\mathrm{Ag}, \mathrm{Hg}$, and $\mathrm{Sn}$ which appear white. The presence of dendrites rich in tin is shown in red and the matrix is cyan which is a $\mathrm{Hg}$ - $\mathrm{Ag}$ combination. All three phases are immediately apparent. This amalgam also contains $\mathrm{Cu}$ which occurs in the same places as does the $\mathrm{Hg}$.

The crystals in this amalgam stand so far above the rest of the surface that optical microscopy is unsatisfactory [2]. No specimen preparation was performed prior to microanalysis. The great depth of focus of the $\mathrm{x}$-ray signal enabled the black-and-whites of figure 7 (See plate d) to be prepared. The color picture serves as a satisfactory "micrograph" of the structure in addition to showing composition effects.

The Tazewell meteorite $(\mathrm{Fe}-16.7 \% \mathrm{Ni})$ is interesting because the iron distribution in the structure constiains the microprobe operator to prepare the $\mathrm{Fe}$ input subject in a special fashion. The meteorite was at one time a single-phase alloy of $\gamma-\mathrm{Fe}-\mathrm{Ni}$ called taenite. As cooling (at a rate of about $2{ }^{\circ} \mathrm{C}$ per million years) proceeded, $\alpha$-Fe called kamacite, was rejected. Finally, at a low enough temperature two-phase $\alpha+\gamma$, called plessite, formed. In addition a number of phosphides of the type $(\mathrm{Fe}-\mathrm{Ni} \mathrm{P})$-called schreibersiteprecipitated out.

The iron weight fraction in kamacite is not much greater than in taenite. Similarly, the Ni level in taenite is not much higher than in plessite; $\mathrm{Ni}$ is absent from kamacite. Thus, the $\mathrm{x}$-ray area for Fe must be taken with the sharpest focus of oscilloscope dot possible (and at low brightness) in order to preserve the structural identity of kamacite. Exposure times are long in this case and the final image is usually gray rather than 
white. Figure 8 shows the results for $\mathrm{Fe}$ and $\mathrm{Ni}$; schreibersite inclusions occur in the kamacite (and probably serve as nucleation sites of kamacite [11]).

The composite must show the kamacite to be nearly pure $\mathrm{Fe}$, the $\mathrm{Ni}$ gradient from taenite into plessite, and
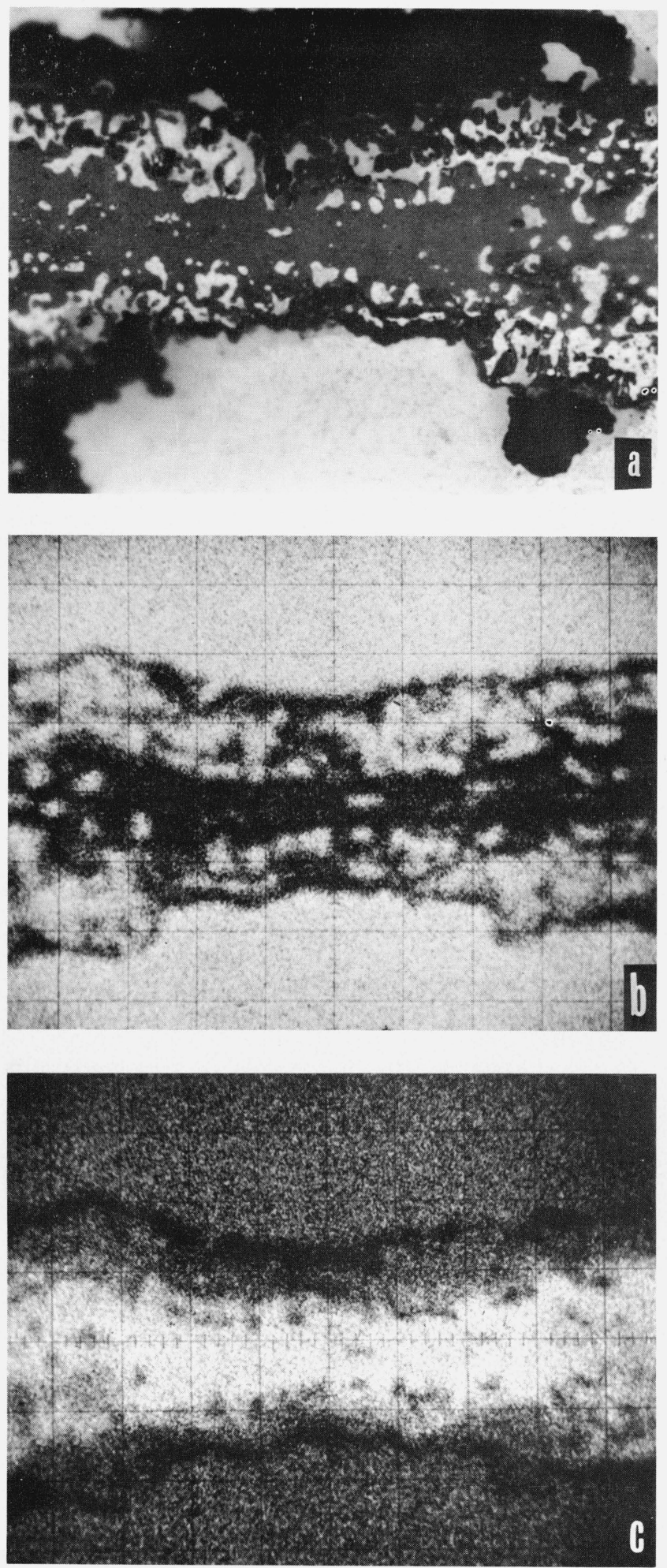

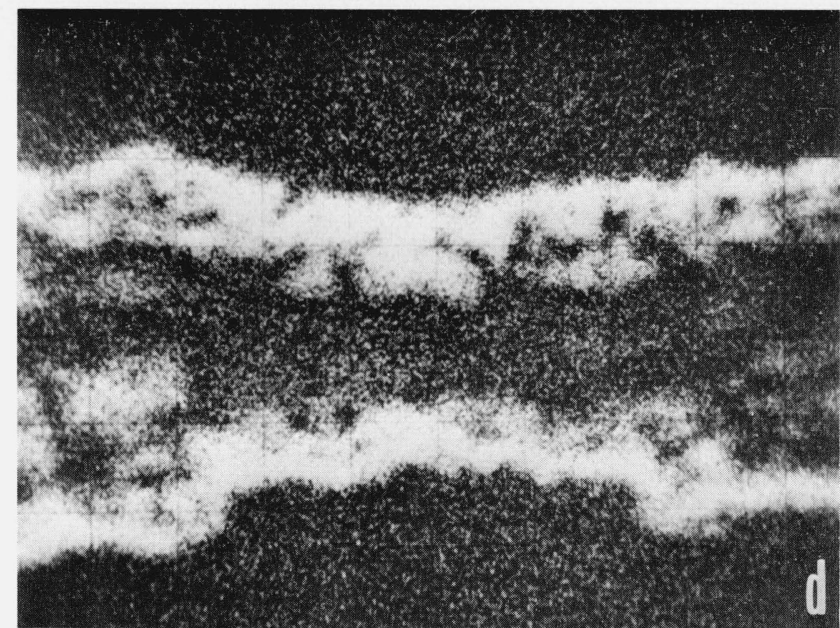

FIGURE 6. Study of a cracked nickel-base superalloy.

a. Optical image (unetched). b. X-ray area scan for $\mathrm{Ni},(\mathrm{K} \alpha$ line).

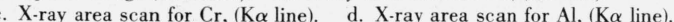

the schreibersite. These features can be seen in figure $8 \mathrm{~d}$, plate 1 . Kamacite is pure red, the $\mathrm{Ni}+\mathrm{Fe}$ of taenite is bright yellow near the kamacite and the yellow intensity decreases going further away from the kamacite and into plessite. Schreibersite is nearly white. The exposure time for red was increased to compensate for the grayness of figure $8 \mathrm{a}$.

A two-color application is shown in figure 9, plate 1 . The specimen is an Amphiuma red blood cell [12]. Phosphorus (red) occurs mainly in the nucleus while sulfur (cyan) is found primarily outside the nucleus. However, regions containing phosphorus are apparent in the periphery (red and white) and, in turn, some sulfur is present in the nucleus as shown by the white regions. White here indicates the superposition of the phosphorus and sulfur signals. The red dots interspersed in the periphery indicate that some phosphorus occurs where there is no sulfur. This tendency is not readily apparent when examining the original blackand-white area scans.

A gold ore serves as a mineralogical application (fig. 10). Several elements are present in the structure. The composite showing $\mathrm{Au}, \mathrm{Si}$, and $\mathrm{Ti}$ is shown in figure $10 \mathrm{~d}$, plate 3 . The ring around the $\mathrm{Si}$ is due to diffusion of x-ray signals and not to $\mathrm{Au}-\mathrm{Si}$ interaction. The input subject for $\mathrm{Si}$ was overexposed. The Ti, Si, and Au superpose at many places; these positions can be easily seen on the composite. The $\mathrm{Ti}$ rich region seems to be a vein in the ore.

Figure 11 (See plate 3 for fig. 11c) shows Cu located in the $\mathrm{Ti}$ rich vein but more finely dispersed than $\mathrm{Ti}$. Backscattered electron signals were used to mark the background.

\section{Conclusion}

Composite color photographs of microprobe scans can be prepared by color-separation methods in a 

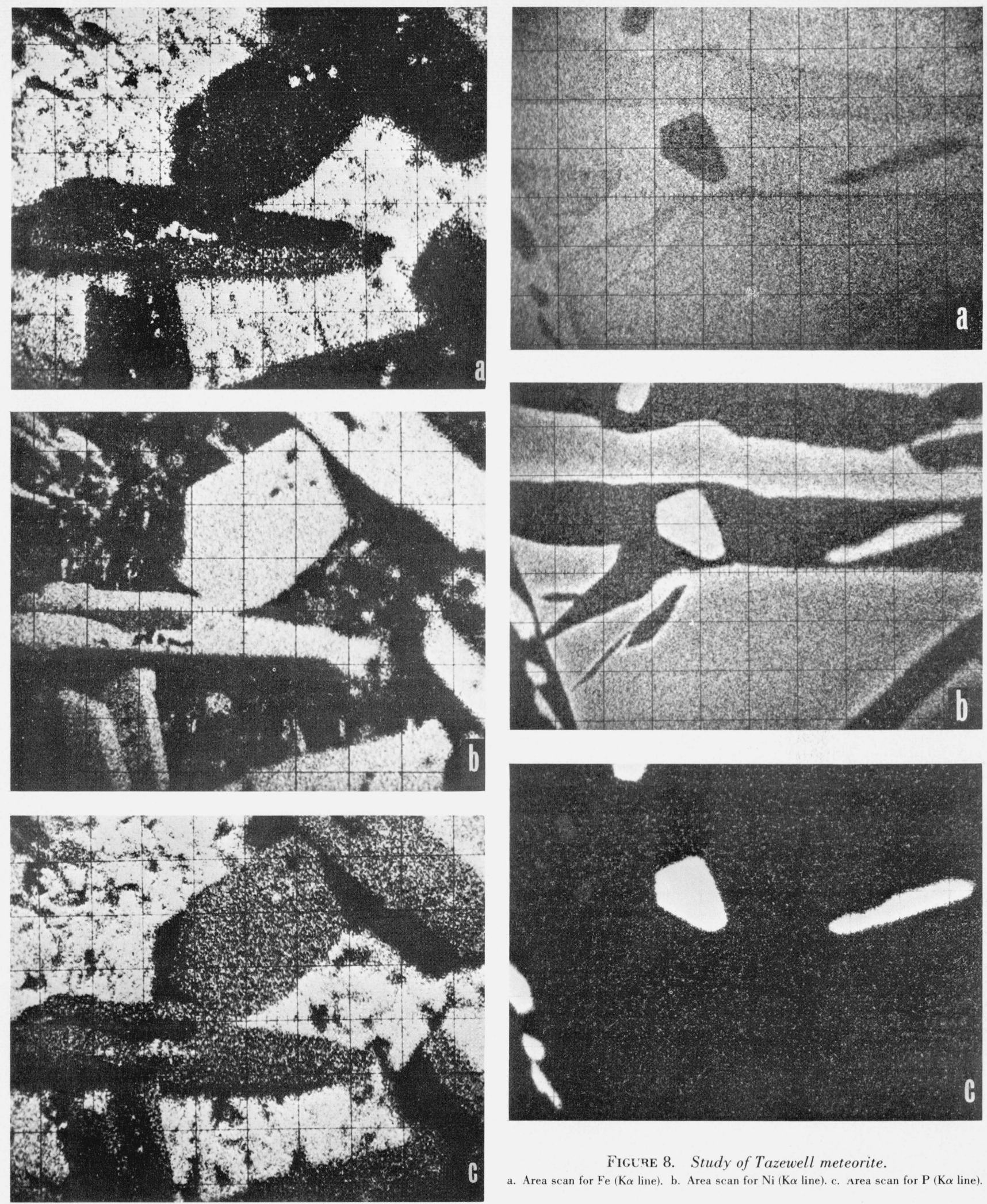

Figure 7. Study of a dental amalgam.

a. Area scan for $\mathrm{Ag},(\mathrm{L} \alpha$ line). b. Area scan for $\mathrm{Sn},(\mathrm{L} \alpha$ line).

Figere 8. Study of Tazewell meteorite.

a. Area scan for $\mathrm{Fe}(\mathrm{K} \alpha$ line). b. Area scan for $\mathrm{Ni}(\mathrm{K} \alpha$ line). c. Area scan for $\mathrm{P}(\mathrm{K} \alpha$ line).

routine fashion. The composites can be readily interpreted in terms of the primary colors representing each element and may be aesthetically pleasing as well. 

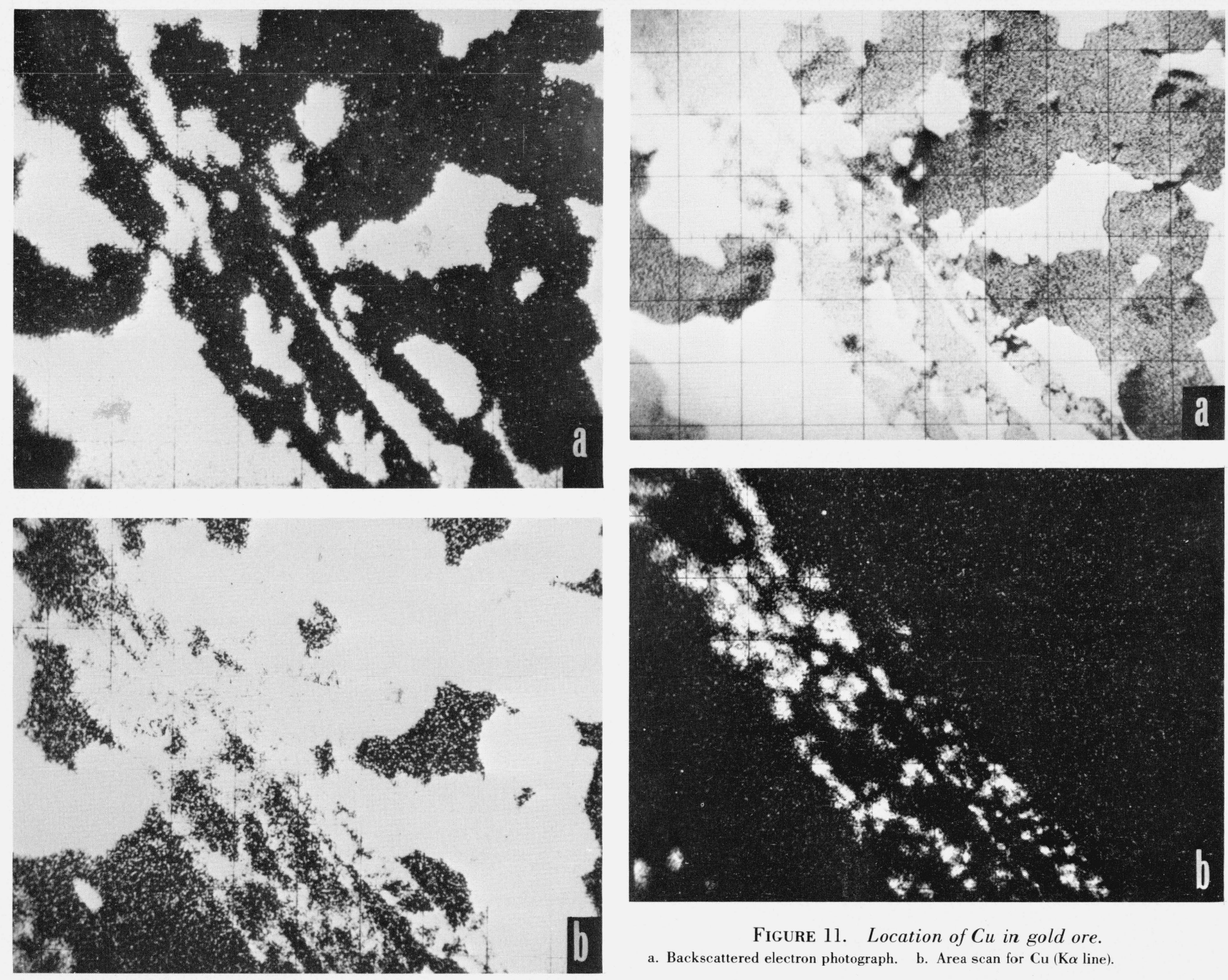

Figure 11. Location of $\mathrm{Cu}$ in gold ore.

a. Backscattered electron photograph. b. Area scan for $\mathrm{Cu}(\mathrm{K} \alpha$ line).

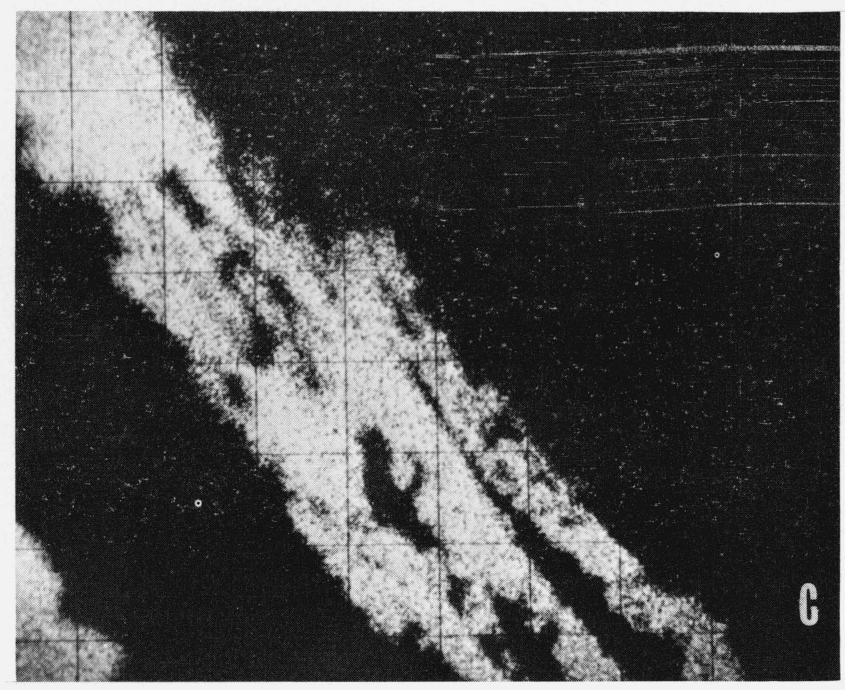

Since the cost of preparing color composites is not prohibitive in time, effort or money, we believe that this method will be accepted as a common means of microprobe data presentation.

\section{References}

[1] Duncumb, P., and Cosslett, V. E., in X-ray Microscopy and Microradiography, p. 347 (V. E. Cosslett, A. Engström and H. H. Pattee, Jr., eds.) (Academic Press, New York (1957)).

[2] Heinrich, K. F. J., ASTM Spec. Tech. Publ. 430, 315 (1968).

[3] Duncumb, P., Op. Cit. p. 617.

[4] Theisen, R., Mem. Sci. Rev. Met. 60, 189 (1963).

[5] Heinrich, K. F. J., Adv. X-ray Anal. 7,382 (1963).

[6] Ficca, J., private communication (1967).

[7] Jones, M. P., J. Gavrilovič, and Beaven, C. H. J., Trans. Inst. Mining and Met. (Sec. B) 75, B274 (1966).

[8] Lennartz, G., and Laffolie, H., Mikrochimica Acta 1967 (Suppl. II) p. 279.

[9] Yakowitz, H., and Heinrich, K. F. J., Metallography 1, No. 1, 55 (1968).

[10] "Color" in Life Magazine for July 3, 1944.

[11] Goldstein, J. I., private communication (1967).

[12] Andersen, C. A., in Methods of Biochemical Analysis 15, 147,

FiguRE 10. Study of gold ore. D. Glick, editor (John Wiley \& Sons, New York, N.Y., 1967).

(Paper 73A2-539) 


\section{Plate 1}

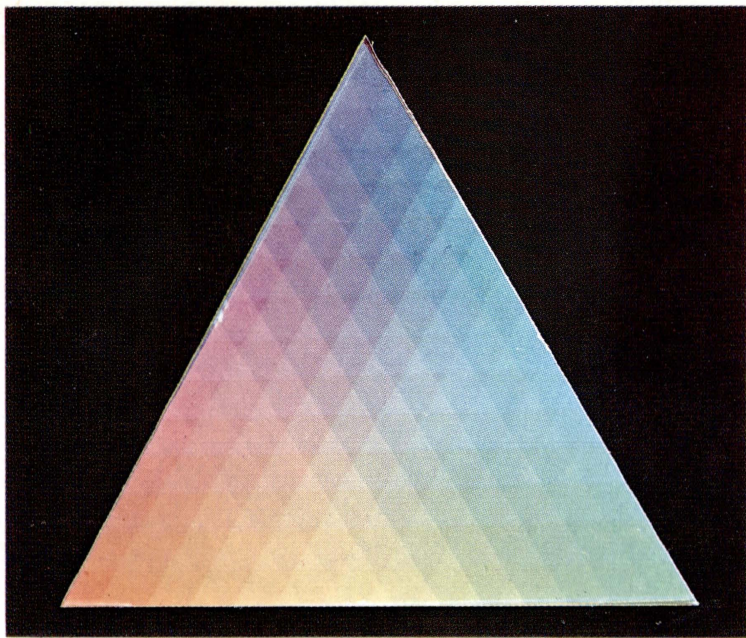

Figure 2. Ternary color mixtures available using rapid development film.

Note grey central region and cyan, magenta, and yellow fields.

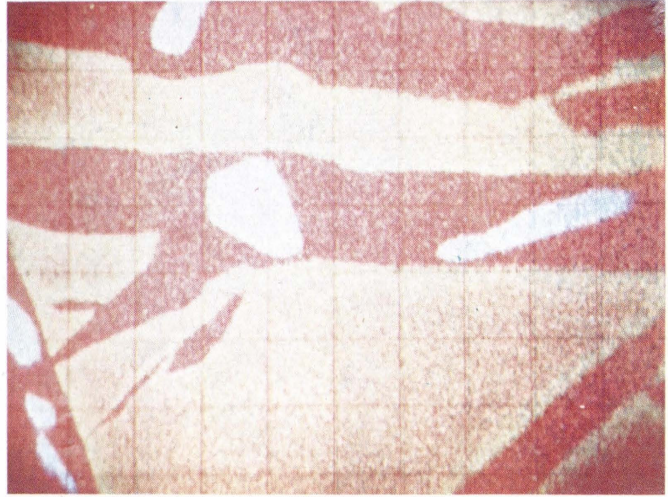

Figure 8d. Composite Fe red, Ni green, $\mathrm{P}$ blue.

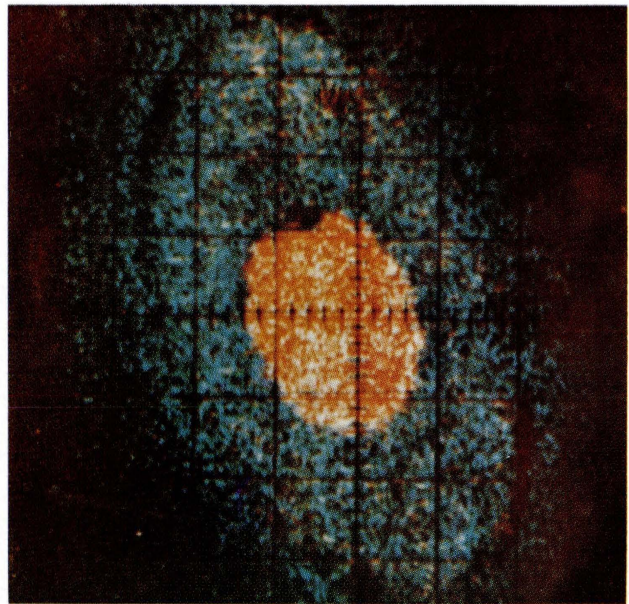

FiguRE 9. Amphiuma red blood cell.

Phosphorus red, sulfur cyan. (Original separation-positives made available through the courtesy of C. A. Andersen, Applied Research Laboratories, Inc., Goleta, Calif.)

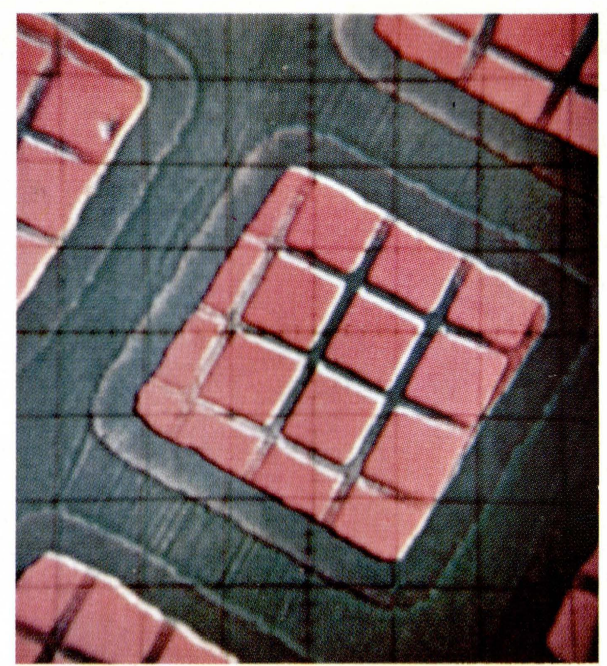

Figure 4. Contrast of each primary and its complement. a. Magenta versus green.

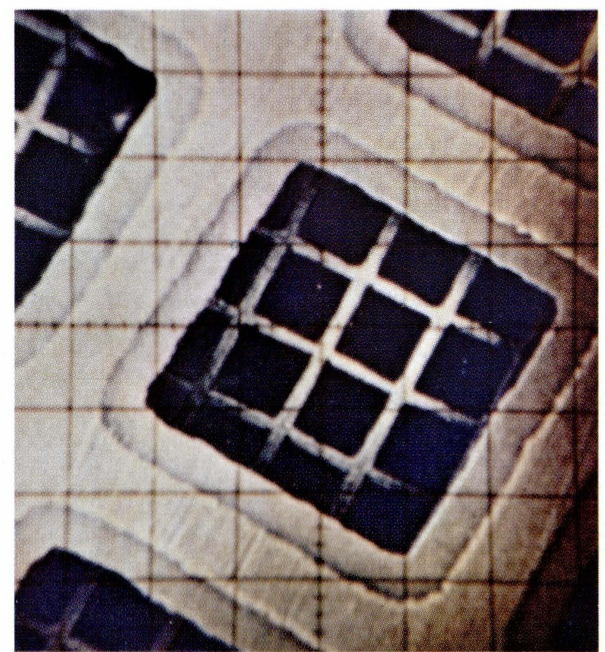

Figure 4b. Yellow versus blue. Note poor yellow-white contrast.

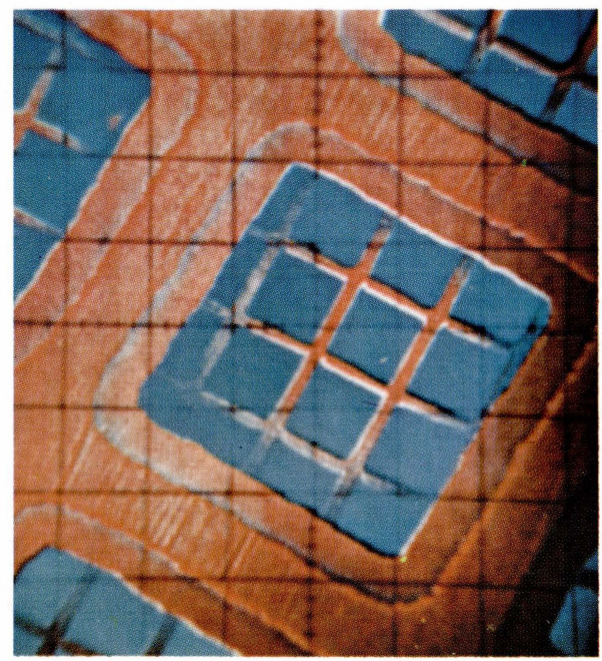

Figure 4c. Cyan versus red. 


\section{Plate 2}

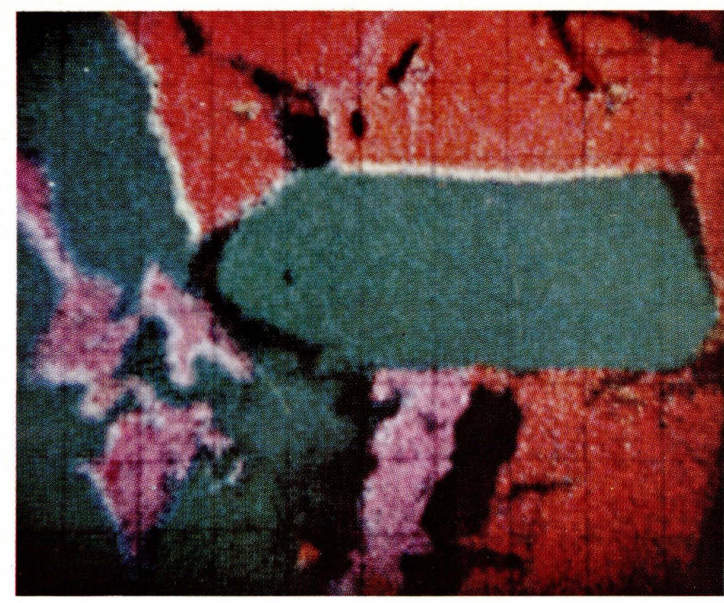

Figure 5d. Si green, Ag blue, S red-Si-S contrast good; Ag-S superposition has fair contrast against $\mathrm{S}$; background suppressed.

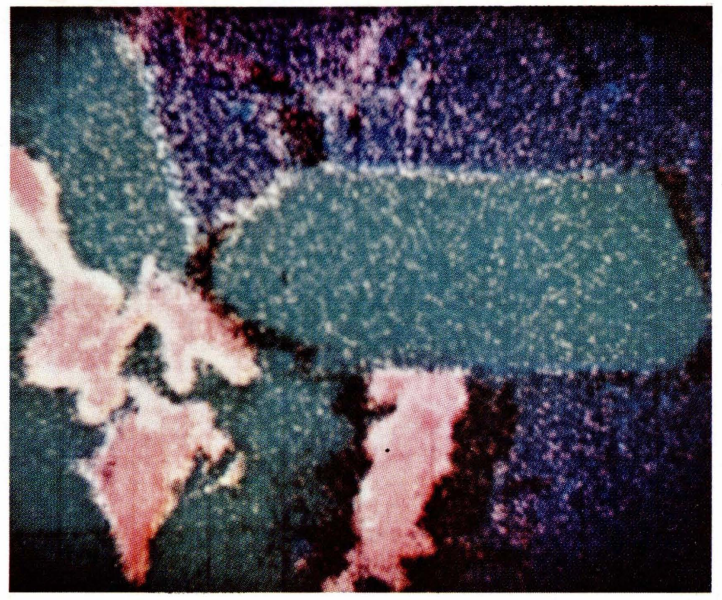

Figure 5e. Si green, Ag red, S blue-Si-S combination poorly contrasted; Ag-S juxtaposition stands out; background prominent.

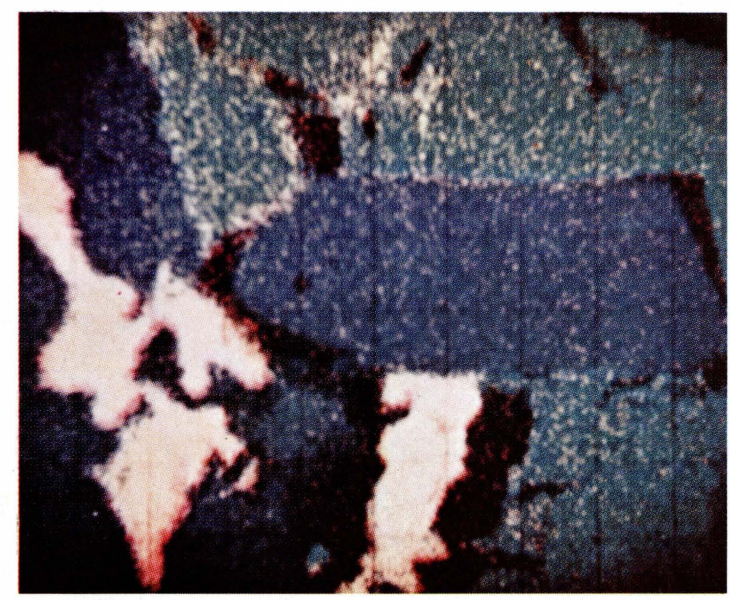

Figure 5f. Si blue, Ag red, S green-Si-S contrast poor; Ag-S superposition stands out; background visible.

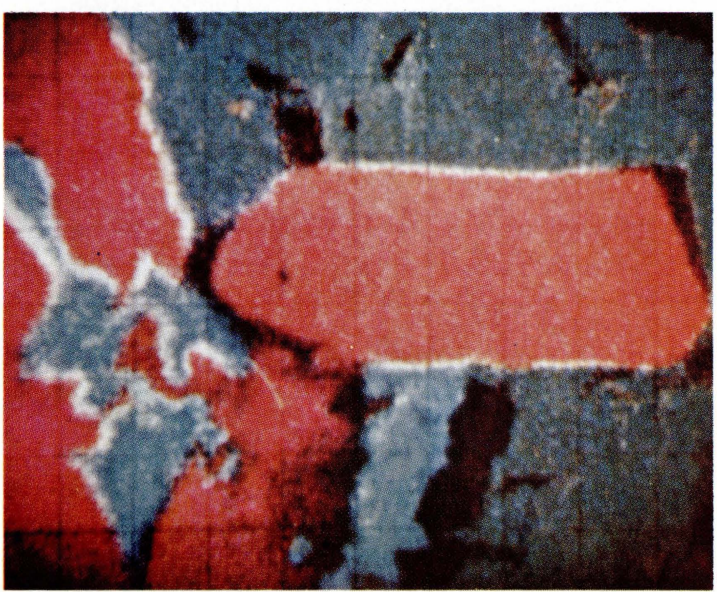

Figure 5h. Si red, Ag blue, S green-Si stands out; Ag-S superposition poorly contrasted with $\mathrm{S}$; background suppressed.

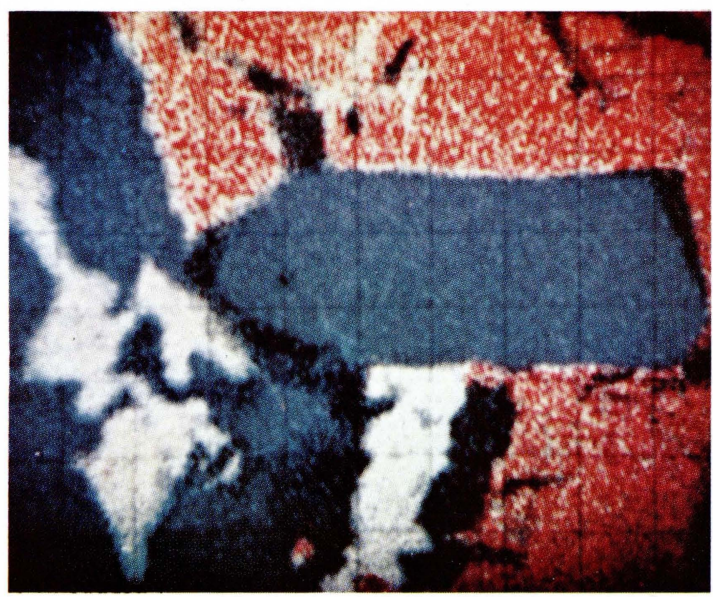

Figure 5g. Si Blue, Ag green, S red-Si-S contrast good; Ag-S superposition stands out; background prominent.

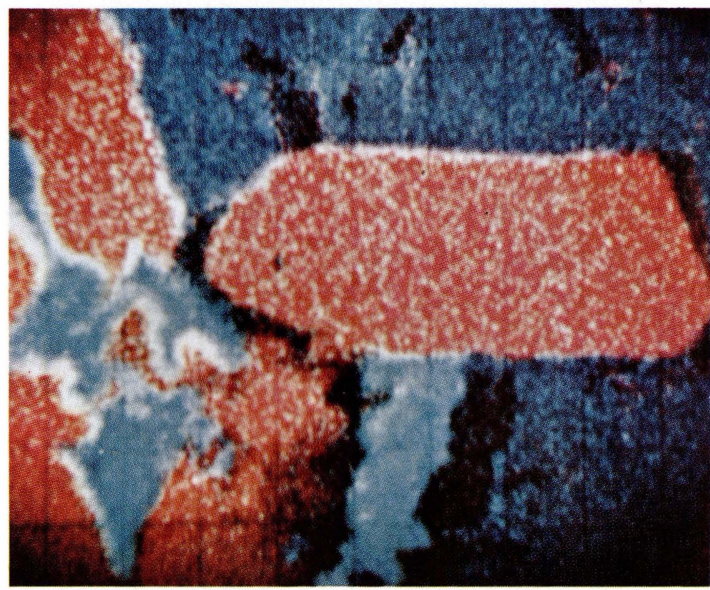

Figure 5i. Si red, Ag green, S blue-Si stands out; Ag-S superposition poorly contrasted with $\mathrm{S}$; background prominent. 


\section{Plate 3}

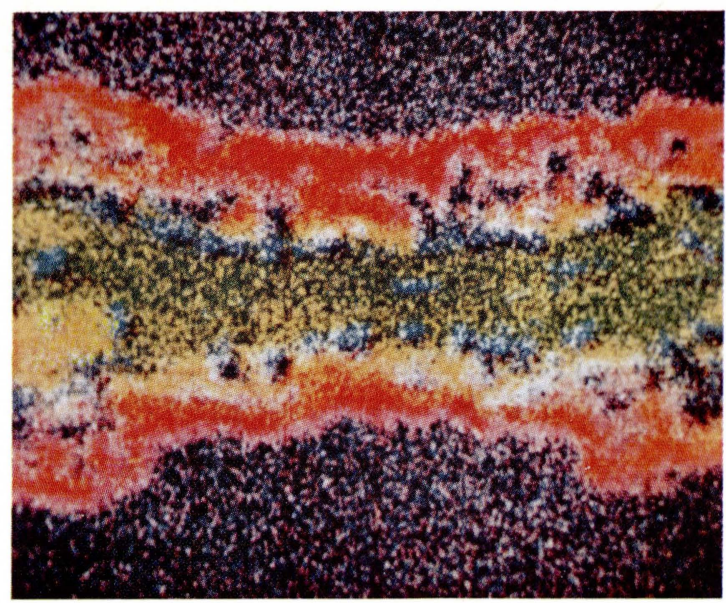

Figure 6e. Composite Ni blue, Cr green, and $\mathrm{Al}$ red.

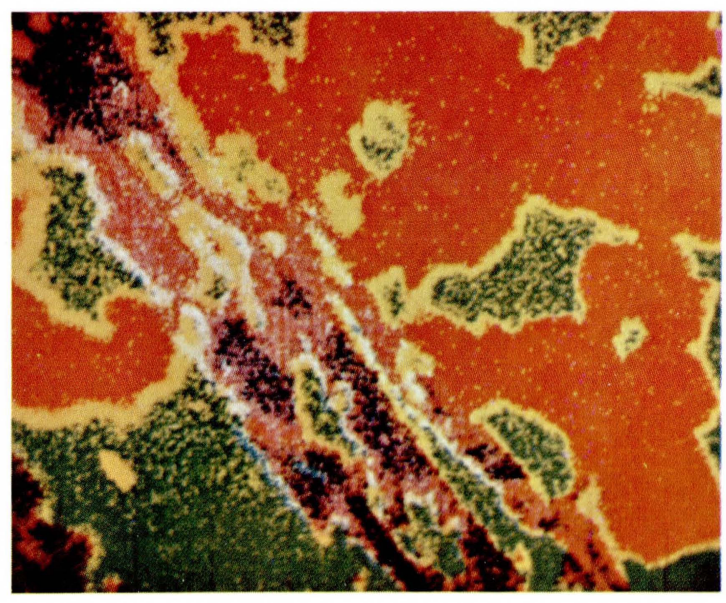

Figure 10d. Composite Au green, Si red, Ti blue.

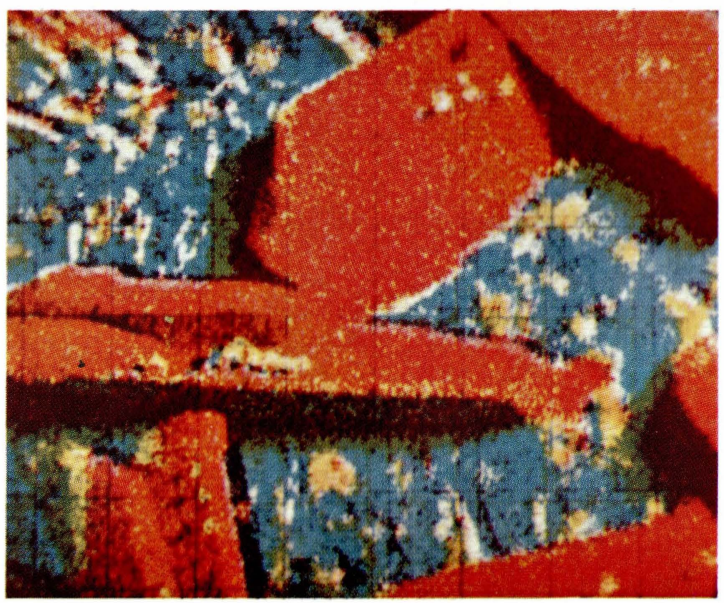

Figure 7d. Composite Ag green, Sn red, Hg blue.

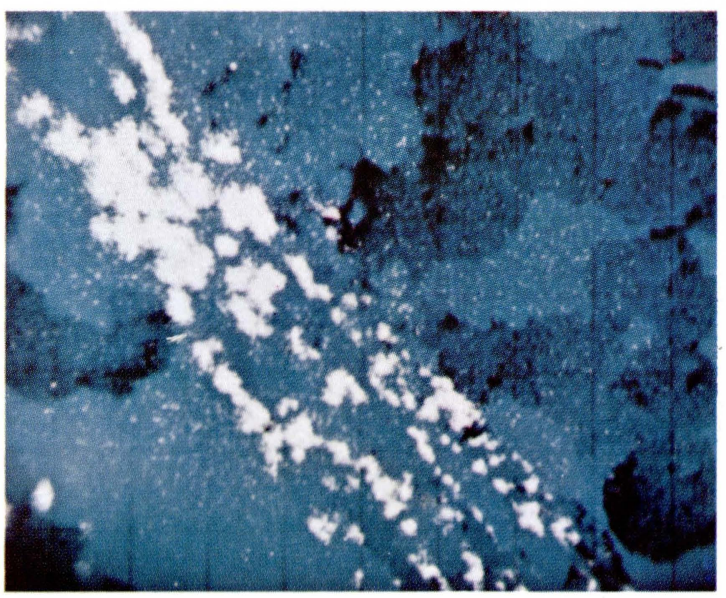

FigURE 1lc. Cu-K x-ray signal white, backscattered electron picture showing the structure is cyan. 